\title{
El desarrollo de la investigación científica en los centros regionales: caso de Unah - Tec Danlí
}

Raúl Orlando Figueroa ${ }^{1}$

Águeda Chávez G. ${ }^{2}$

\section{A. Fundamentación normativa de la investigación}

Los cambios manifiestos en los pilares de docencia, vinculación e investigación en la Universidad Nacional Autónoma de Honduras (UNAH) no son producto de la casualidad ni la improvisación, ya que obedece a la fundamentación y acuerdos internacionales que se han llevado a cabo en cumbres de carácter normativo respecto a la educación superior. Para el caso, con las conferencias mundiales sobre la educación superior, en el año 1998 y posteriormente en el 2009 celebradas en París, se comienza la instrumentalización a través de un marco de acción que contempla misiones y funciones. La Declaración Mundial sobre Educación Superior en el siglo XXI se adopta desde la Unesco, declaración que fue discutida y elaborada por distintas regiones del mundo, incluyendo América Latina. Esto forma parte de los sustentos teóricos para un replanteamiento de la investigación en la institución comprendido entre el 2006 a 2017.

Entre las interrogantes enunciadas en estas conferencias mundiales tenemos: ¿Estamos promoviendo la investigación en todas las disciplinas, comprendidas las ciencias sociales y humanas y las artes, dada su pertinencia para el desarrollo humano sostenible? ¿Han asumido nuestras universidades la investigación sobre la problemática de la educación superior y del sistema educativo, como una de sus tareas prioritarias? Interrogantes clave y pertinentes para diferentes momentos, pues el conocimiento es cambiante, y es necesario saber si está siendo promovido de acuerdo a las exigencias y necesidades de la sociedad; esto solamente se puede saber con indagación, la cual debe ser realizada por expertos que genera la misma Universidad.

\footnotetext{
${ }^{1}$ Ph. D. en Ciencias de la Cultura Física, Master en Educación, Licenciado en Cultura Física, Coordinador Regional de Investigación de la UNAH-TEC-Danlí. Pitolamaquina2@hotmail.com

2 Máster en enseñanza del español como lengua extranjera. Máster en Lexicografía Hispánica por la Universidad de León, España, la Asociación de Academias de la Lengua Española (ASALE) y la RAE. Miembro de la Unidad de Investigación de Humanidades en UNAH- Tec Danlí. agueda.chavez@unah.edu.hn
} 
Con el argumento que se generó en 1998 por la Unesco y posteriormente en 2009, donde se menciona que las instituciones de educación superior deben adoptar estructuras organizativas y estrategias educativas que les confieran un alto grado de agilidad y flexibilidad, al respecto consideramos que la actual Dirección de Investigación Científica y Posgrados (DICYP) representa esa unidad académica, pues lo anterior a ella es argumento teórico. En la práctica, después de los once años de la Reforma de la UNAH y, muy específicamente, en la investigación, se ofrece de forma concreta y tangible esta adopción, la cual incluye la transformación desde varios puntos de vista (cualitativos y cuantitativos), pues se carecía de una unidad: visión, misión, reglamentos, valores, estructura y demás componentes dignos de una máxima casa de estudio.

En el Plan General para la Reforma Integral de la UNAH se enuncia un aspecto, que consideramos muy acertado: "Solamente si la UNAH produce un cambio profundo en su quehacer académico y de investigación aplicada, entonces la fuentes de ingresos se incentivarán, incluso la posibilidad de fondos adicionales que pudiera proporcionar el Estado" (2005:30). Y es que solo de esa manera la población en general confiaría, como confía ahora en la UNAH y en sus diferentes unidades académicas. La institución se ha posicionado en rankings a nivel Latinoamericano y del Caribe, a nivel general y de la investigación con una reforma planificada con visiones, misiones, objetivos estratégicos, indicadores de impacto y resultados esperados generando procesos que se desarrollan paulatinamente, cumpliendo lo planteado.

La UNAH ha asumido el compromiso con la sociedad del conocimiento otorgado por ley en la Constitución de la República (Art. 160), de esta manera somos corresponsables con todos los sectores involucrados en la transformación de la sociedad. De los tres ejes fundamentales en torno a lo que gira el quehacer universitario, es la investigación el mecanismo en el que gira el pilar de la universidad del presente siglo, el cual se convierte a la vez en un desafío para el docente y la institución misma, en cuanto a la suma de esfuerzos humanos y financieros se trata. Este pilar, reglamentado en la Ley Orgánica de la Universidad Nacional Autónoma de Honduras en su artículo 102 enuncia que:

La Investigación constituye una de las funciones esenciales, es el medio que sirve, tanto de fundamento y retroalimentación, que es el instrumento base para la creación y transferencia social del conocimiento para la docencia como motor para el progreso de la comunidad nacional, por lo que debe ser pertinente para el desarrollo del país y la Institución a nivel Nacional como Regional (Acuerdo No. 206-07 p. 128) 
Estos aspectos determinan las líneas que deben ser transversalizadas en los currículos de las diferentes carreras ofrecidas. En este sentido la institución tiene muy claro que la investigación amerita de un plan estratégico, para ello articula el desarrollo de la investigación en ejes prioritarios.

\section{B. De la estructura administrativa en UNAH- TEC Danlí}

El licenciado Elio Altamirano, Historiador y profesor jubilado de la UNAH (37 años de trabajo en la UNAH) comenta que hace una década la investigación científica no estaba organizada ni descentralizada, que eran unas pocas facultades las que investigaban, así como el poco número de investigadores para los que no existía una base de datos que los registrara. (E. Osorio Altamirano, comunicación personal, 08 de junio de 2016). También sustenta que los centros regionales universitarios antes de la Reforma Universitaria brillaban por su ausencia, y que es muy reciente el hecho de estar dando resultados, al igual que otras facultades, escuelas, departamentos y secciones de Ciudad Universitaria.

Leticia Salomón, actual directora de la DICYP, también es del criterio que antes de su gestión la descentralización era necesaria, por lo que a su llegada, a esta dirección, creó la estructura de la Coordinación Regional de Investigación la cual tendría una especie de función de la Dirección de Investigación Científica de la UNAH (DICU) en los centros regionales universitarios y en la zona de acción.

Esta decisión de Salomón ha sido de lo más acertada, pues se refleja en la participación de las diferentes facultades y centros regionales universitarios, tal es el caso del Centro Regional Universitario Sur-Oriental (UNAH- TEC Danlí) que suma un número importante de ponencias desde el segundo congreso. Este centro regional universitario, se ha adjudicado becas básicas y sustantivas, investigación como asignación académica, y también importantes premios de investigación científica en sus diferentes categorías. Siendo el benjamín de los centros regionales universitarios de la mano de la Reforma Universitaria y de la DICYP ha superado las expectativas que sobre él se tenían.

Desde este centro nos apuntamos para alcanzar la excelencia en todos los pilares a través de la formación profesional de los docentes con estudios de maestrías y doctorados. Actualmente se cuenta con 28 magísteres y dos docentes con grado de doctorado; 11 docentes cursan maestrías en los diferentes campos del saber (en la modalidad presencial como a distancia); cuatro estudian doctorados: dos en Chile, uno en Nicaragua y uno en Honduras. El docente de este siglo debe ser capaz de compren- 
der el creciente rol de su papel y que él es la clave para transformar el pensamiento desde la educación a través del conocimiento, que este no se consigue únicamente con el grado - este es solo el inicio-, posteriormente deberá lanzarse en su búsqueda. Actualmente el monopolio del saber, que antes y durante mucho tiempo ostentó los sistemas de enseñanza formal a través del Estado, se ha desarticulado con la puesta en órbita de la tecnología. Esta misma tecnología se ha vuelto herramienta fundamental para el docente, de manera que ahora tiene una doble preocupación: hacerse del conocimiento y compartirlo. A pesar de la percepción que se tiene de una sociedad con alto acceso a la información computarizada, el rol del docente no ha perdido vigencia, no obstante, se cuestiona con preocupación su desempeño actual. En este sentido, las buenas prácticas del conocimiento adquirido son fundamentales.

La rigurosidad científica es el tratamiento formal de un proceso intelectual reflejado en un problema, el cual debe ser analizado de forma severa implementando los métodos más apropiados. Es por ello que es necesario, en la investigación científica, poseer las competencias necesarias para poder afrontar los cambios que se manifiestan en el mundo actual del conocimiento. Las maestrías académicas son un punto esencial para crear competencias y así generar cambios significativos en esta área del conocimiento, sin dejar atrás las que generan los doctorados y los post doctorados. La UNAH a través de la DICYP viene diseñando un camino para los docentes desde hace 11 años a través de capacitaciones que doten de competencias para afrontar las exigencias que se da la actualidad en el campo de la investigación, enseñando las diferentes normativas que se requieren en las diferentes disciplinas de publicación a nivel local, nacional e internacional.

UNAH TEC Danlí está estructurado con los mínimos planteamientos dentro de la normativa de la DICYP: Consejo General de Investigación -CGI-, Coordinación Regional, siete unidades de investigación, un grupo de investigación multidisciplinario compuesto por las unidades de Humanidades, Ciencias de la Salud y Ciencias Económicas bajo las cuales se organiza toda la actividad investigadora relativa a cada eje. Tal como se refleja en el esquema siguiente: 


\section{Estructura administrativa de la Investigación en la UNAH-TEC-Danlí}

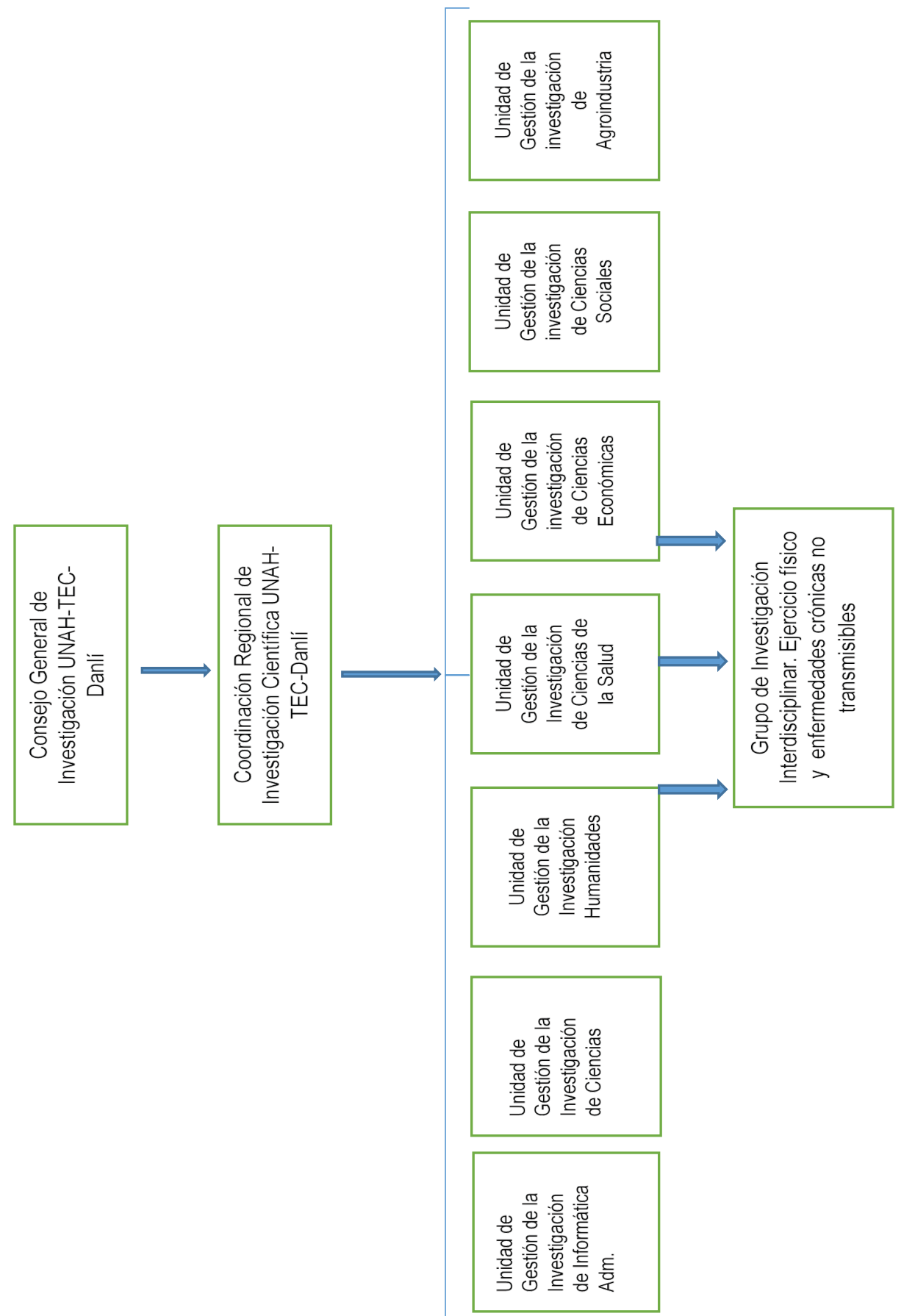




\section{Resultados y reconocimientos}

Priorizamos las líneas de investigación sobre las que la universidad propone aunar esfuerzos, ya que la profundización en los conocimientos en cada uno de los 13 ejes establecidos representa una herramienta valiosa para la compresión, interpretación y posterior aporte a la realidad nacional. De acuerdo a las investigaciones registradas tenemos que se destaca el eje de la salud, seguido de la seguridad alimentaria y los demás ejes. Algunas de estas investigaciones han sido interdisciplinares, por lo que también se busca conformar más equipos de investigación para fortalecer los demás ejes. Por otro lado, desarrollamos una investigación aplicada y útil para la sociedad, relevante y visible con alcance internacional. Por último, vinculamos dicha investigación a la docencia, de manera que acercamos a nuestros estudiantes los procesos de la investigación científica en su campo, para que conozcan de primera mano los últimos avances y resultados de la misma, este es el caso de la Jornada de Investigación Científica UNAH- TEC Danlí que se realiza durante tres días cada año, a finales de noviembre en donde participan los docentes y estudiantes involucrados.

Algunos artículos producto de las investigaciones de las diferentes Unidades de Investigación han sido publicados en revistas nacionales e internacionales, créditos otorgados en libros institucionales y en capítulos de libro. Si bien el número de profesores que han publicado libros es bajo aún, únicamente cuatro, no deja de ser relevante para estos fines ya que dan muestra del aporte significativo al conocimiento desde un centro novísimo (gráfico 3). Los libros publicados son:

1. Así de sencillo... se aprende Voleibol. Raúl Orlando Figueroa

2. Lecturas de Juegos Organizados. Raúl Orlando Figueroa.

3. Seminario de Investigación Científica. Raúl Orlando Figueroa.

4. Historia de las bebidas, embriaguez y alcoholismo en Honduras. Julio Sevilla

5. El Impronunciable nombre de Dios. Julio Sevilla

6. Manual de Ortografía: La hora de escribir bien. Águeda Chávez G.

7. Pensamiento variacional emergente. Marvin Mendoza.

8. Matemáticas. Marvin Mendoza.

Las ponencias realizadas a través de convocatorias para participar en eventos de gran alcance desde congresos, simposios y seminarios han sido presentadas

\footnotetext{
${ }^{3}$ Marvin Mendoza, Julio Sevilla, Águeda Chávez G. y Raúl Orlando Figueroa.

${ }^{4}$ Algunos de los libros se han realizado en colaboración con otros autores de las disciplinas en cuestión
} 
utilizando las diferentes técnicas: mesas de trabajo, paneles, mesa redonda y conferencias. Las realizadas a nivel internacional en Cuba, México, Guatemala, Costa Rica, Alemania y España ${ }^{5}$ son producto de las investigaciones desde los estudios de maestría, doctorado y de las becas sustantivas otorgada por la DICYP.

En el gráfico 1 se reflejan las participaciones al Congreso de Investigación desde la UNAH, con un promedio de cinco ponencias por año; gráfico 2 da cuenta de ponencias en otros eventos nacionales y cinco ponencias por año internacionalmente.

\section{Gráfico 1. Participaciones en el CIC- DICYP desde el 2008 a 2016}

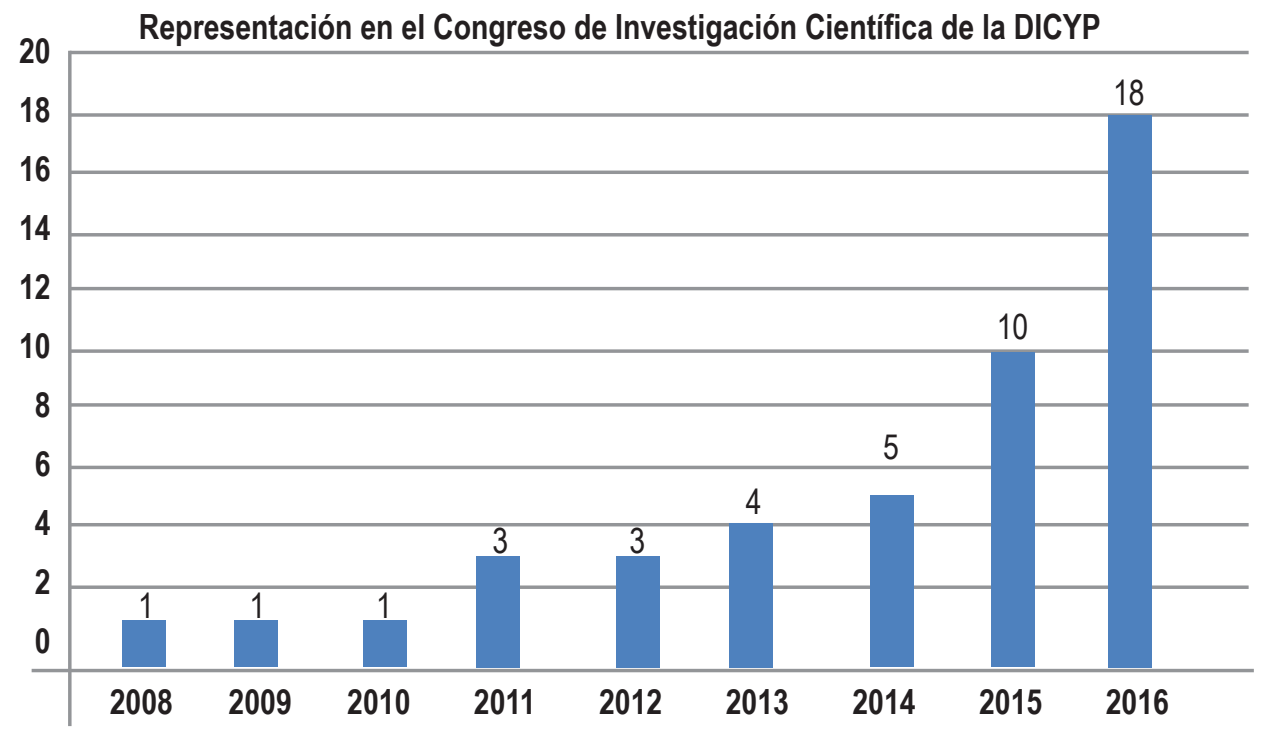

Fuente: Consejo General de Investigación UNAH- TEC Danlí

Para conseguir la rigurosidad metodológica propia de la investigación científica y que la máxima casa de estudios impulsa desde la DICYP nos sumamos a las capacitaciones permanentes que esta ofrece a través de la movilización de nuestros docentes hasta la Ciudad Universitaria o bien sea de los capacitadores hasta el centro regional. Los profesores que han cursado el Diplomado en Investigación Científica suman el 68 por ciento, ubicados en las diferentes áreas del conocimiento que se imparten en el centro regional. El gráfico arroja un número de cuatro docentes por año.

\footnotetext{
${ }^{5}$ Raúl Figueroa, Marvin Mendoza y Jairo Martínez son los docentes que han realizado estas ponencias internacionales.
} 
Gráfico 2. Otras ponencias nacionales e internacionales de 2008 a 2017

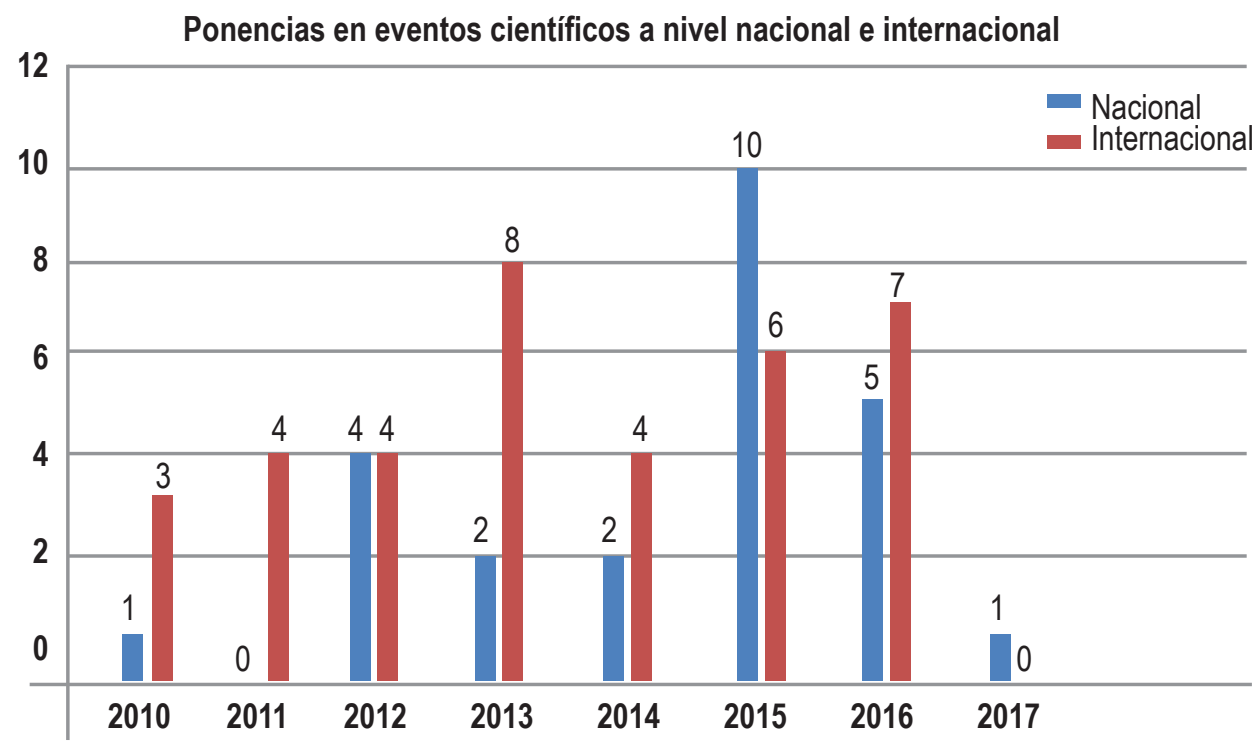

Fuente: Consejo General de Investigación UNAH- TEC Danlí

Gráfico 3. Publicaciones de 2008 a 2017

publicaciones realizadas

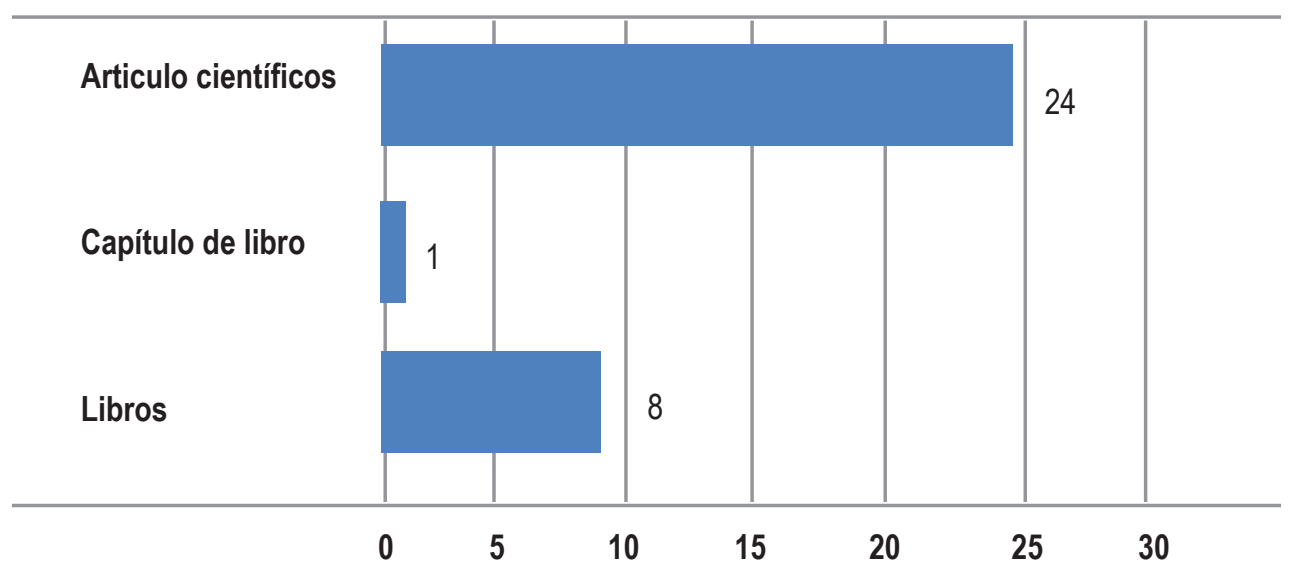

Fuente: Consejo General de Investigación UNAH- TEC Danlí 


\section{Gráfico 4. Capacitación del personal docente desde el 2010}

Participaci[on de docentes en cursos y el diplomado (DICYP)

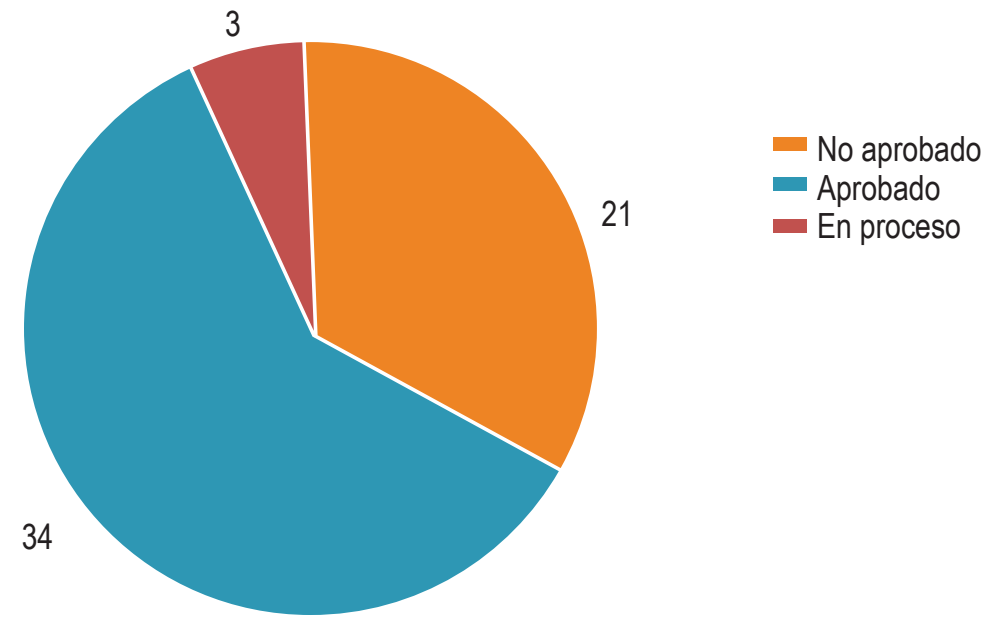

Fuente: Consejo General de Investigación UNAH- TEC Danlí

\section{Reconocimientos obtenidos}

Las becas y reconocimientos otorgados han sido ganados a pulso, pues no son más que fruto del trabajo y de la confianza que desde el CGIC de este centro se les proyecta a los docentes, puesto que estos son a través de convocatorias abiertas a nivel nacional mediante bases concretas que persiguen, financiar o destacar las actividades y/o resultados de investigación que generan aportes significativos al desarrollo científico y tecnológico.

Hemos obtenido lo siguiente:

- 1 becas sustantiva.

- 8 becas básicas de investigación.

- 2 becas para profesores egresados de posgrado.

- 1 beca para estudiantes de grado.

- $\quad 11$ becas por carga académica.

- 4 premios de investigación. (Ganado 2 de Ideas de innovación y tecnología, 1 en Investigador en formación a nivel profesional y 1 en excelencia en la investigación científica a la mejora de la calidad de vida). 
En el 2013, el premio a la categoría de Ideas de innovación tecnológica lo obtuvo Fredy Torres de la Carrera de Agroindustria; para el 2014, Enrique Alberto Molina, de Informática Administrativa lo obtiene a la misma categoría; en el 2015, Raúl Orlando Figueroa Soriano, a la categoría Investigador en formación a nivel profesional y para el 2016, el premio obtenido por Raúl Orlando Figueroa Soriano, Miriam Ordóñez y el Jaime Valerio es para la categoría Excelencia en Investigación a la mejora de la calidad de vida. Es importante mencionar que para la convocatoria del 2017 se han realizado tres postulaciones.

\section{CONCLUSIONES}

Sabemos que debemos ir mejorando de acuerdo a las exigencias y las implicaciones de un proceso constante, tal como se ha observado a lo largo de esta década de existencia. La institución seguirá por la senda trazada, de manera que esperamos que quienes en un futuro la integren, conozcan y sigan la brecha de los que positivamente han forjado un centro líder a nivel nacional de forma holística, y muy específicamente, en el campo investigativo. Sabemos que también es necesario continuar las capacitaciones, así aumentaremos el nivel investigativo de la unidades académicas para hacer propuestas para los rediseños de los planes de estudio de los grados que lo ameriten e insertar el componente investigación como un aspecto transversal. Esa transversalización en un futuro podría ser una de las alternativas que conllevaría a crear el andamiaje sobre el conocimiento de la investigación, que posteriormente su utilización ayudaría al profesional a aprender a enseñar.

\section{BIBLIOGRAFÍA}

Consejo General de Investigación de Unah Tec Danlí (2017) Encuentro Anual de Coordinadores Regionales de Investigación año 2017. [Diapositivas de Power Point]Consultado en los Archivos del CGI/ Unah- Tec Danlí

La educación superior en América Latina y el Caribe: diez años después de la Conferencia Mundial de 1998. (2008) Editor, Carlos Tünnermann Bernheim. - Cali: lesalc-Unesco, PUJ

Ley Orgánica de la Universidad Nacional Autónoma de Honduras. Acuerdo No. 206-07 (2007). Recuperado de: http://www.poderjudicial.gob.hn/CEDIJ/Leyes/- 
Documents/Dec462013ReformaArt8AdicionLeyUNAH.pdf

Plan General para la Reforma Integral de la UNAH. Recuperado de: http://Plan-General-para-la-Reforma-Integral-de-la-UNAH.pdf

Unesco. (1998) Conferencia mundial sobre la educación superior. La educación superior en el siglo XXI: Visión y acción. Recuperado de: http://www.mineducacion.gov.co/1759/articles230245_archivo_pdf_conferencia_cmes.pdf 